\title{
Herz- und Gefäßschutz durch inkretinbasierte Therapie?
}

\begin{abstract}
Für GLP-(Glucagon-Like-Peptide-)1 sowie für inkretinbasierte Therapien gab es tierexperimentell und in Pilotstudien Hinweise auf kardiovaskulär protektive Effekte. Ergebnisse entsprechender Endpunktstudien werden mit Spannung erwartet.
\end{abstract}

„Seit Werner Creutzfeldt im Jahr 1979 die enteroinsuläre Achse beschrieb, wurde eine Vielzahl weiterer Effekte des Inkretins GLP-1 aufgedeckt", sagte Prof. HansUlrich Häring von der Universität Tübingen. Neben seinen vielfältigen Effekten auf den Glukose- und Fettstoffwechsel, vermittelt über Rezeptoren in Pankreas, Fettgewebe, Magen, Leber und Gehirn, hat GLP-1 vermutlich auch eine direkte antiatherosklerotische Wirkung auf die Gefäßwände. So wiesen schwedische Wissenschaftler bei Diabetikern eine endothelabhängige Vasodilatation durch GLP-1 nach. In Kardiomyozyten steigert
GLP-1 die Produktion von cAMP (zyklisches Adenosin-Mono-Phosphat) und wirkt damit positiv chronotrop. Gleichzeitig hemmt es dort die Apoptose.

\section{Verbesserte Funktion des linken Herzventrikels mit GLP-1}

In einer nicht randomisierten Pilotstudie erhielten Patienten mit akutem Myokardinfarkt nach Rekanalisierung eine 72-stündige Infusion mit GLP-1. Darunter verbesserte sich die linksventrikuläre Funktion (ejection fraction) von durchschnittlich 30 auf $40 \%$ und somit signifikant gegenüber der Kontrollgruppe.
In einer anderen Pilotstudie verbesserte sich unter GLP-1 die kardiale Belastbarkeit bei Patienten mit einer Herzinsuffizienz der NYHA-(New York Heart Association-)Klassen III und IV.

„Auch bei den neuen inkretinbasierten Antidiabetika gibt es eine Reihe von Hinweisen auf möglicherweise protektive kardiovaskuläre Effekte“, berichtete Prof. Nikolaus Marx von der Universitätsklinik Aachen. Der seit etwa fünf Jahren in der Behandlung bei Typ-2-Diabetes zugelassene DPP-4-Hemmer Sitagliptin (etwa als Januvia ${ }^{\circledR}$ im Handel) reduzierte den Makrophagen- und erhöhte den Kollagengehalt in Atheroskleroseplaques von Apolipoprotein-E-Knockout-Mäusen.

„Ob sich diese viel versprechenden Ergebnisse in eine Verminderung kardiovaskulärer Ereignisse übersetzen, werden wir in einigen Jahren aus den laufenden Endpunktstudien mit inkretinbasierten Medikamenten erfahren“, so Marx.

Bericht: Thomas Bißwanger-Heim, Freiburg

Quelle: Symposium „GLP-1 360“” am 16.5.2012 bei der DDG-Jahrestagung in Stuttgart, Veranstalter: MSD

\section{Warnsystem informiert über gefährliche Blutzuckermuster}

\author{
Um das Risiko für Hyper- und Hypoglykämien und damit Folgekompli- \\ kationen des Diabetes zu senken, sind regelmäßige und häufige Blut- \\ zuckermessungen eine sinnvolle Option. Ein innovatives Messsystem \\ macht Patienten auf anomale Blutzuckermuster aufmerksam und er- \\ möglicht innen so eine bessere metabolische Kontrolle als bisher.
}

Leitliniengemäß wird heute beim Diabetes-Management eine normnahe $\mathrm{HbA}_{1 c^{-}}$ Einstellung gewünscht, um das Risiko für diabetische Folgekomplikationen möglichst gering zu halten. Je strikter aber die Blutzuckereinstellung, umso größer die Gefahr von Unterzuckerungen, die ihrerseits wieder Komplikationen nach sich ziehen. Die Empfehlung für Diabetiker lautet daher, an zwei bis drei aufeinanderfolgenden Tagen nach Mustern im niedrigen oder hohen Blutzuckerbereich zu suchen, um eine möglichst stabile Blutzuckereinstellung zu erreichen. Das Erkennen solch pathologischer Blut- zuckermuster wird jetzt durch ein neues Gerät erheblich vereinfacht: Das OneTouch $^{\star}$ Verio $^{\circ}$ IQ-System wurde gezielt für Patienten mit Typ-1- oder Typ-2-Diabetes entwickelt, die ihre Insulindosis Tag für Tag in Abhängigkeit von Ernährung und sportlicher Aktivität selbst anpassen, was ein gewisses Hypoglykämierisiko mit sich bringt. Als einziges Blutzuckermessgerät besitzt das neue Gerät die Verio ${ }^{\circ}$ PatternAlert ${ }^{\text {tx }}$ Technologie, mit deren Hilfe relevante Abweichungen in den Blutzuckerwerten aufgedeckt werden. Bei jeder Messung sucht das neue System automatisch nach hohen und niedrigen Blutzuckertrends und informiert den Patienten über Auffälligkeiten.

Ein hoher Blutzuckertrend ist gegeben, wenn innerhalb der gleichen Zeitspanne von drei Stunden dreimal hohe präprandiale Werte $(130 \mathrm{mg} / \mathrm{dl})$ während der vergangenen fünf Tage gemessen werden. Die Warnung für einen niedrigen Blutzuckertrend erfolgt bei zwei niedrigen Werten innerhalb des gleichen Intervalls von drei Stunden in den letzten fünf Tagen. Warnmeldungen über zu niedrige Werte werden blau, die über zu hohe Werte rot angegeben.

Weitere Annehmlichkeiten des neuen Messsystems sind das beleuchtete Farbdisplay mit hoher Auflösung, das Messungen auch bei ungünstigem Licht erlaubt. Anja Renfordt, mehrfache Weltmeisterin im Kickboxen und Typ-1-Diabetikerin, freut sich zudem über die Schnelligkeit der Messung, was die Blutzuckerbestimmung im Berufs- und Trainingsalltag oft erst ermöglicht.

Bericht: Dr. Katharina Arnheim, Freiburg

Quelle: Mediatalk „OneTouch ${ }^{\circledast}$ Verio ${ }^{\oplus}$ IQ" bei der DDG-Jahrestagung am 17.5.2012 in Stuttgart, Veranstalter: LifeScan 